\title{
Análisis in silico de lipocalinas de perro, gato, caballo, vaca, hámster y gallina. Posible efecto en el estudio de las enfermedades alérgicas
}

Andrés Sánchez ${ }^{1-3}$, Ricardo Cardona ${ }^{3}$, Jorge Sánchez ${ }^{3,4}$

\section{Resumen}

ANTECEDENTES: Ias lipocalinas parecen explicar la reactividad cruzada entre animales como gato y perro, pero poco se ha estudiado acerca de su papel en la reactividad cruzada con otros animales y su efecto clínico.

OBJETIVOS: analizar por técnicas bioinformáticas la identidad entre lipocalinas de diferentes especies y explorar la utilidad de estas técnicas en el estudio de las alergias.

MATERIAL Y MÉTODO: estudio in silico en el que se buscaron las secuencias de lipocalinas utilizando el programa BLAST. Las secuencias de proteínas se alinearon con el programa CLUSTAL Omega versión 1.2.1 de UniProt. Las secuencias base de los alineamientos fueron las lipocalinas de perros y gatos. La identidad entre las lipocalinas se comparó con la frecuencia de sensibilización a animales en una población de 284 pacientes alérgicos.

RESULTADOS: las secuencias mostraron identidades entre 10 y $70 \%$. Los valores más altos se encontraron entre Can f 6-Fel d 4 (68\%) y Fel d 4-Equ c 1 (68\%). La identidad más baja fue con las lipocalinas purpurina y proteína de unión al retinol del gallo (menor de 20\%). Observamos una relación entre el patrón de sensibilización y el grado de identidad entre las especies estudiadas.

CONCLUSIONES: a partir de los estudios bioinformáticos y los patrones de sensibilización encontrados se propone que Fel d 4 y Equ c 1 son posibles alergenos mayores para gato y caballo en la población del trópico y comparten alta reactividad cruzada con Can $\mathrm{f} 6$. Debido a que estos resultados provienen de modelos predictivos, deben confirmarse con estudios in vitro e in vivo.

PALABRAS CLAVE: alergia, aves, bioinformática, caballo, gato, gallina, identidad, lipocalinas, mascotas, sensibilización.

\begin{abstract}
1 Universidad del Magdalena, Departamento de Medicina, Santa Marta, Colombia.

${ }^{2}$ Corporación Universitaria Rafael Núñez, Programa de Medicina, Cartagena, Colombia.

${ }^{3}$ Grupo de Alergología Clínica y Experimental. IPS Universitaria, Universidad de Antioquia, Medellín, Colombia.

${ }^{4}$ Grupo de Alergología Experimental e Inmunogenética, Instituto de Investigaciones Inmunológicas, Universidad de Cartagena, Cartagena, Colombia.
\end{abstract}

Recibido: 25 de mayo 2015

Aceptado: 27 de noviembre 2015

Correspondencia

Dr. Jorge Sánchez

jotamsc@yahoo.com

Este artículo debe citarse como

Sánchez A, Cardona R, Sánchez J. Análisis in silico de lipocalinas de perro, gato, caballo, vaca, hámster y gallina. Posible efecto en el estudio de las enfermedades alérgicas. Rev Alerg Méx. 2016 ene-mar;63(1):1-10. 


\section{In silico analysis of the identity of lipocalin of dog, cat, horse, cow, hamster and hen. Possible role in allergic diseases.}

\author{
Andrés Sánchez ${ }^{1-3}$, Ricardo Cardona ${ }^{3}$, Jorge Sánchez ${ }^{4}$
}

\begin{abstract}
BACKGROUND: Lipocalins seem to explain the cross-reactivity between some pets such as cat and dog. However, its role in other animals and its possible clinical impact in allergy diseases have been scarcely studied.
\end{abstract}

OBJECTIVE: To analyze by bioinformatics techniques, the identity between lipocalin of some animals and to explore the clinical impact on allergic diseases.

MATERIAL AND METHOD: An in silico study was done to search for lipocalin sequences using the BLAST program of NCBI Database. The protein sequences were aligned with CLUSTAL Omega UniProt version 1.2.1 software. The base sequences for alignments were lipocalins dogs and cats. The defined percentage identity was compared with the frequency of sensitization to animals exposed in a population of 284 patients with suspected allergic diseases.

RESULTS: Identities between sequences were $10 \%$ to $70 \%$. The highest values were found with Can f 6-Fel d 4 (68\%) and Fel d 4-Equ c 1 $(68 \%)$. The lower identity was found with lipocalin porpurin and retinol binding $(<20 \%)$. We observed a relationship between sensitization and the percent identity between the species studied.

CONCLUSIONS: Lipocalins as Can f 6, Fel de 4 and Equ c 1 seem to play an important role in the cross-reactivity to cat, horse and dog but not for the co-sensitization to hamster, cow or birds. Fel de 4 and Equ c 1 could be a prevalent allergen for horse and cat. These results come from predictive analysis and must be confirmed by in vitro and in vivo studies.

KEYWORDS: allergy; birds; bioinformatics; horse; cat; hen; identity; lipocalin; pets; sensitization

\begin{abstract}
${ }^{1}$ Universidad de Magdalena, Departamento de Medicina, Santa Marta, Colombia.

${ }^{2}$ Corporación Universitaria Rafael Núñez, Programa de Medicina, Cartagena, Colombia. ${ }^{3}$ Grupo de Alergología Clínica y Experimental. IPS Universitaria, Universidad de Antioquia, Medellín, Colombia.

${ }^{4}$ Grupo de Alergología Experimental e Inmunogenética, Instituto de Investigaciones Inmunológicas, Universidad de Cartagena, Cartagena, Colombia.
\end{abstract}

Correspondence

Dr. Jorge Sánchez

jotamsc@yahoo.com

\section{ANTECEDENTES}

La frecuencia de atopia y enfermedades alérgicas, como asma, rinitis y conjuntivitis es alta y parece que la incidencia está aumentando especialmente en los países industrializados. ${ }^{1}$
Entre las principales fuentes de alergenos están las mascotas; existe un número creciente de familias en Latinoamérica con mascotas en sus casas. Si la exposición prolongada a mascotas es un factor de riesgo de, o protector contra, la aparición de alergias es un tema aún en discusión; 
sin embargo, entre los pacientes con sospecha de alergias, las guías médicas recomiendan realizar las medidas de evitación, ${ }^{2}$ pero por diferentes motivos, entre ellos emocionales, la mayoría de los pacientes tiene dificultades para conseguir este objetivo; además, la efectividad clínica de esta recomendación no es clara. ${ }^{3}$

Un problema adicional para lograr las medidas de evitación es la reactividad cruzada entre algunas mascotas, especialmente entre gatos y perros, que son las mascotas que con más frecuencia generan alergias en Europa. ${ }^{4}$ En estudios previos observamos que la sensibilización a mascotas también es prevalente en algunas ciudades de Latinoamérica. ${ }^{5}$ Las lipocalinas son proteínas que transportan pequeñas moléculas, como esteroides, retinoides y lípidos. Esta familia de proteínas está presente en la mayor parte de los mamíferos, al igual que en bacterias, nematodos e insectos, y son alergenos importantes como causa de la reactividad cruzada entre caballo, gato y perro. Sin embargo, poco se sabe acerca del papel de las lipocalinas en la sensibilización a otras fuentes comunes en Latinoamérica.

Los análisis bioinformáticos, entre los que se incluyen los análisis in silico (simulación por computadoras), son una herramienta útil para el modelamiento y la predicción de la estructura de las proteínas. Estas herramientas pueden ayudar a predecir alergenos mayores potenciales y pueden explicar la reactividad cruzada entre dos o más fuentes de alergenos. Si en una población la frecuencia de sensibilización a varias fuentes es conocida, los análisis de alineamiento y la identidad entre las proteínas pueden ser el primer paso para identificar los alergenos principales que explican los patrones de sensibilización y cosensibilización entre las especies. Posteriormente, es necesario demostrar si los alergenos identificados como probables son realmente alergenos mayores mediante estudios in vivo. En este estudio exploramos por medio de técnicas bioinformáticas la identidad entre las lipocalinas de perro, gato, caballo, vaca, hámster y gallina y su posible papel en las alergias a animales en la población del trópico.

\section{MATERIAL Y MÉTODO}

\section{Secuencias de lipocalinas}

Estudio in silico en el que se buscaron lipocalinas utilizando el programa BLAST de la base $\mathrm{NCBI}$, con las secuencias validadas hasta el 20 de noviembre de 2014. Sólo se incluyeron las proteínas alergénicas con una identificación completa de la secuencia de ARN mensajero de perro, gato, caballo, hámster y vaca. Además, se incluyeron dos lipocalinas de gallina no identificadas como alergénicas (purpurina y proteína de unión a retinol: RBP) para tener secuencias comparativas de este grupo taxonómico. Las entradas de secuencias parciales o cortes alternativos se excluyeron. Algunas lipocalinas potencialmente alergénicas, como Equ c 2, no se incluyeron en este estudio debido a que la secuencia reportada en la base NCBI es incompleta.

Las secuencias proteicas se alinearon usando el programa CRUSTAL Omega, versión 1.2.1. de UniProt. Las secuencias base para los alineamientos fueron las lipocalinas de perro y gato, debido a que son las mascotas más comunes en las casas.

\section{Análisis filogenéticos}

Los análisis filogenéticos basados en las secuencias de ARN (11 secuencias de nucleótidos) se realizaron mediante el método de reconstrucción de Neighbor-Joining con el programa MEGA (molecular evolutionary genetics analysis) versión 6, usando el método bootstrap como prueba de filogenia con 500 replicaciones. Para determinar la distancia filogenética utilizamos el método de la máxima probabilidad compuesta, teniendo en cuenta el número de sustituciones de base 
por cada sitio. Todas las posiciones con espacios vacíos se eliminaron (deleciones completas). Para todos los cruces, las posiciones de codón incluidas para los análisis fueron primero+segun do+tercero+secuencias no codificantes. Debido al número de secuencias de lipocalinas utilizadas no realizamos subanálisis filogenéticos.

\section{Selección de la población}

Usamos una cohorte de pacientes previamente reportada ${ }^{5}$ y agregamos pacientes nuevos reclutados durante el periodo de junio de 2012 a marzo de 2014. Recolectamos 284 pacientes con diagnóstico de asma, rinitis o dermatitis atópica que asistieron al servicio de Alergología Clínica de la Universidad de Antioquia (Medellín, Colombia) y requirieron una prueba cutánea con alergenos para identificar los posibles alergenos ambientales. Durante la cita para la prueba se interrogó a los pacientes acerca de los detonantes sospechosos y la existencia de mascotas en casa o la exposición indirecta a animales. Luego de la prueba cutánea, los pacientes recibieron información de manera verbal y por escrito acerca de las medidas de control ambiental para las fuentes a las que estuvieron sensibilizados.

Como un criterio de inclusión, a todos los pacientes se les evaluó la sensibilización a Blomia tropicalis, Dermatophagoides pteronyssinus, Dermatophagoides farinae, epitelio de gato y perro. Incluimos extractos adicionales de animales en los pacientes con contacto o con sospecha de síntomas por otros animales (por ejemplo, caballo, hámster, pájaros, gallinas, etc.). El diagnóstico se estableció de acuerdo con las guías GINA de asma (www.ginasthma.org), ARIA de rinitis, ${ }^{6}$ el Consenso Latinoamericano de Conjuntivitis $^{7}$ y los criterios de Hanifin y Rajka de dermatitis atópica. ${ }^{8}$

Para la prueba cutánea intraepidérmica usamos los extractos estandarizados de los laboratorios
Leti e Inmunotek (Madrid, España) y las recomendaciones internacionales para su lectura (habón $3 \mathrm{~mm}$ mayor que el control negativo). ${ }^{4,9}$ Todos los pacientes suspendieron la administración de antihistamínicos u otros fármacos el tiempo requerido antes de la prueba.

\section{Consideraciones éticas}

Para la revisión de las historias clínicas y los datos de los pacientes se obtuvo la aprobación del comité de ética de la institución hospitalaria. Las secuencias se tomaron de la base de datos de NCBI, por lo que son de acceso abierto.

\section{Análisis estadísticos}

Los análisis se realizaron con el programa IBM SPSS versión 21 para Windows. Los resultados se expresaron como porcentajes de frecuencia y en números absolutos. Se usaron análisis de correlación, prueba de $\chi^{2}$ o regresiones logísticas multivariables para medir las diferencias entre los grupos.

\section{RESULTADOS}

\section{Identidad de las lipocalinas}

Las lipocalinas seleccionadas se muestran en el Cuadro 1. Se realizaron 38 alineamientos entre las lipocalinas incluidas. Las secuencias bases utilizadas fueron las lipocalinas de perro y gato (Cuadro 2). Las secuencias mostraron una gran variabilidad entre las identidades (10 a $70 \%$ ). La mayor identidad se encontró entre los alineamientos de Can $\mathrm{f} 6$-Fel d 4 con valores de $68 \%$ y Fel d 4 -Equ c 1 con $68 \%$, seguido por Can f 1 -Fel d 7 con 61\% y Can f 6-Equ c 1 con $58 \%$ (Figura 1). La menor identidad se encontró entre los alineamientos para porpurina y RBP con las otras especies, que fue menor de $20 \%$ (Cuadro 3 y 4 ). 
Cuadro 1. Lipocalinas seleccionadas para los alineamientos

\begin{tabular}{|c|c|c|c|c|}
\hline Lipocalina & Código & $\begin{array}{c}\text { Base de datos } \\
\text { (secuencia ARNm) }\end{array}$ & $\begin{array}{c}\text { Entrada } \\
\text { (Swissprot) }\end{array}$ & $\begin{array}{l}\text { Número de } \\
\text { aminoácidos }\end{array}$ \\
\hline \multicolumn{5}{|c|}{ Perro (Canis familiaris) } \\
\hline Can $\mathrm{f} 1$ & ALL1_CANFA & AF027177 & 0.18873 & 174 \\
\hline Can $\mathrm{f} 2$ & ALL2_CANFA & AF027178 & 0.18874 & 180 \\
\hline Can $\mathrm{f} 4$ & D7PBH4_CANFA & GU132996 & D7PBH4 & 174 \\
\hline Can $\mathrm{f} 6$ & H2B3G5_CANFA & HE653774 & H2B3G5 & 190 \\
\hline \multicolumn{5}{|c|}{ Gato (Felis domesticus o catus) } \\
\hline Fel d 4 & ALL4_FELCA & AY497902 & Q5VFH6 & 186 \\
\hline Fel d 7 & E5D2Z5_FELCA & GU108332 & E5D2Z5 & 180 \\
\hline \multicolumn{5}{|c|}{ Bovine (Bos taurus) } \\
\hline Bos d 2 & ALL2_BOVIN & L42867 & Q28133 & 172 \\
\hline \multicolumn{5}{|c|}{ Caballo (Equus caballus) } \\
\hline Equ c 1 & ALL1_HORSE & U70823 & Q95182 & 187 \\
\hline \multicolumn{5}{|c|}{ Gallina/gallo (Gallus gallus o domesticus) } \\
\hline Purpurina & PURP_CHICK & M17538 & P08938 & 196 \\
\hline RBP & RET4_CHICK & X77960 & P41263 & 196 \\
\hline \multicolumn{5}{|c|}{ Hámster (Phodopus sungorus) } \\
\hline Pho s 21 kDa & S5ZYD3_PHOSU & KF148615 & S5ZYD3 & 151 \\
\hline
\end{tabular}

Lipocalinas seleccionadas de diferentes animales para análisis bioinformáticos. RBP: proteína de unión al retinol (retinol binding proteins).

Cuadro 2. Identidad entre las lipocalinas de perro y gato

\begin{tabular}{lccc}
\hline Alineamiento & $\begin{array}{c}\text { Identidad } \\
(\%)\end{array}$ & $\begin{array}{c}\text { Núm. de } \\
\text { aminoácidos } \\
\text { idénticos }\end{array}$ & $\begin{array}{c}\text { Núm. de } \\
\text { aminoácidos } \\
\text { similares }\end{array}$ \\
\hline \multicolumn{4}{c}{ Perro-gato } \\
Can f 1-Fel d 4 & 23 & 43 & 61 \\
Can f 1-Fel d 7 & 61 & 110 & 46 \\
Can f 2-Fel d 4 & 25 & 48 & 68 \\
Can f 2-Fel d 7 & 24 & 45 & 64 \\
Can f 4-Fel d 4 & 25 & 48 & 66 \\
Can f 4-Fel d 7 & 19 & 37 & 50 \\
Can f 6-Fel d 4 & 68 & 130 & 38 \\
Can f 6-Fel d 7 & 23 & 43 & 62 \\
\hline
\end{tabular}

Con la lipocalina de hámster (Pho s 21 kDa), la identidad con otras especies también fue baja: Can f 4-Pho s 21 kDa: 25\%. Fel d 7-Pho s 21 kDa: $17 \%$. La lipocalina de la vaca Bos d 2 mostró valores entre 18 y $30 \%$, la mayor identidad se encontró con Can f 4 con un valor de $31 \%$ (Cuadros 3 y 4 ).

\section{Análisis filogenéticos}

El árbol óptimo con una longitud de ramificación igual a 5.00589491 se muestra en la Figura 2. El porcentaje de árboles replicados (500 repeticiones) se muestra en la Figura 2. El árbol se muestra a escala, la longitud de la ramificación representa la distancia evolutiva en la cantidad de cambios acumulados; sin embargo, se debe tener en cuenta que el método Neighbor-Joining sólo hace agrupaciones de "miembros similares", sin tomar en cuenta el reloj molecular, por lo que la distancia evolutiva es sólo aproximada de acuerdo con la identidad de las proteínas estudias. Hubo 411 posiciones en la base final.

\section{Características de la población y sensibilización a animales}

De los 284 pacientes, 149 eran mujeres (Cuadros 5 y 6). La edad media fue de 20 años. La mayoría de los pacientes tenían enfermedades respiratorias 


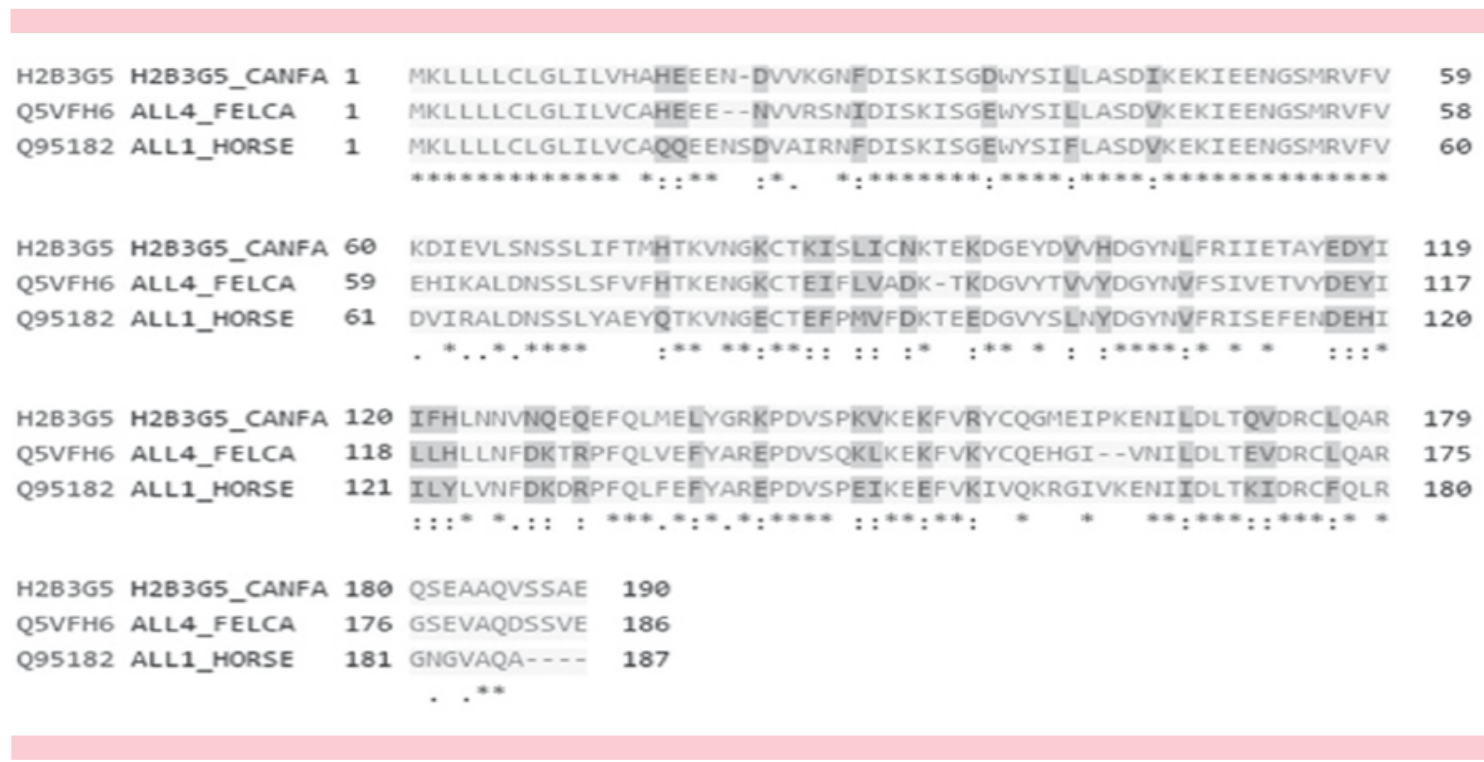

Figura 1. Estructura de alineamiento entre Can f 6, Fel d 4 y Equ c 1. Los aminoácidos identificados están indicados con "*", los aminoácidos similares están indicados con ":" y resaltados en gris oscuro.

(90\%) y 38 pacientes (13\%) tenían síntomas respiratorios y cutáneos; 227 pacientes (80\%) tenían atopia, 151 pacientes (53\%) fueron positivos a uno $o$ varios animales. El perro $(n=136,48 \%)$ y el gato $(\mathrm{n}=27,9.5 \%)$ fueron las fuentes causantes con más frecuencia de sensibilización entre mascotas; 18 pacientes positivos a gato lo fueron también a perro. Seis pacientes fueron positivos a caballo y todos ellos lo fueron a gato y perro. Cuatro de ellos tenían una alta exposición a caballo; 33 (14\%) pacientes fueron positivos a pájaros, 24 de ellos fueron positivos a gato o perro; 135 pacientes positivos a perro (99\%) resultaron sensibilizados a ácaros y todos los pacientes positivos a gato lo fueron a los ácaros; 219 (77\%) pacientes estuvieron expuestos a algún animal: $133(46 \%)$ a perro, $86(30 \%)$ a gato, 70 (24\%) a pájaros y $33(11 \%)$ a otros animales, como caballo, hámster y conejo. No pudimos evaluar si existía una relación entre la exposición a mascotas y los síntomas, debido a que la población estaba conformada totalmente por pacientes alérgicos y no había controles. La única asociación entre exposición y sensibilización fue a pájaros $(\mathrm{p}<0.1$, media: 2.8, IC 95\%: 1.3-6).

\section{Relación entre identidad de lipocalinas y patrón de sensibilización}

Observamos que el porcentaje de identidad de algunas lipocalinas está estrechamente relacionado con el patrón de sensibilización. La mayor identidad se encontró para Can f 6, Fel d 4 y Equ c 1, con frecuencia alta de cosensibilización a perro, gato y caballo ( $p$ menor de 0.05). Las lipocalinas de gallina, hámster y vaca tenían poca identidad y poca frecuencia de cosensibilización con gato y perro. Debido a que esta asociación podría estar relacionada con el grado de exposición, realizamos un subanálisis, considerando la exposición animal como una covariable, y no observamos una relación significativa entre la exposición directa a caballo, hámster, vaca, gallina, gato o perro y sensibilización.

\section{DISCUSIÓN}

Los patrones de sensibilización pueden variar de acuerdo con las condiciones geográficas y socioculturales de una región. ${ }^{10-12}$ De acuerdo 
Cuadro 3. Identidad entre lipocalinas usando las secuencias del perro como base

\begin{tabular}{|c|c|c|c|}
\hline Alineamiento & $\begin{array}{c}\text { Identidad } \\
(\%)\end{array}$ & $\begin{array}{l}\text { Núm. de } \\
\text { aminoácidos } \\
\text { idénticos }\end{array}$ & $\begin{array}{l}\text { Núm. de } \\
\text { aminoácidos } \\
\text { similares }\end{array}$ \\
\hline \multicolumn{4}{|c|}{ Caballo-bovino } \\
\hline Can $\mathrm{f} 1$-Bos d 2 & 19 & 35 & 57 \\
\hline Can $\mathrm{f} 2$-Bos d 2 & 18 & 34 & 65 \\
\hline Can $\mathrm{f}$ 4-Bos d 2 & 31 & 54 & 56 \\
\hline Can $\mathrm{f}$ 6-Bos d 2 & 25 & 47 & 63 \\
\hline \multicolumn{4}{|c|}{ Perro-caballo } \\
\hline Can f 1-Equ c 1 & 25 & 46 & 59 \\
\hline Can f 2-Equ c 1 & 26 & 49 & 70 \\
\hline Can f 4-Equ c 1 & 27 & 52 & 63 \\
\hline Can f 6-Equ c 1 & 58. & 111 & 48 \\
\hline \multicolumn{4}{|c|}{ Perro-gallina } \\
\hline Can $\mathrm{f} 1$-Purpurin & 13 & 28 & 57 \\
\hline Can f 1-PUR & 14 & 29 & 63 \\
\hline Can $\mathrm{f}$ 2-Purpurin & 13 & 28 & 59 \\
\hline Can $\mathrm{f}$ 2-PUR & 17 & 35 & 66 \\
\hline Can $\mathrm{f}$ 4-Purpurin & 13 & 28 & 46 \\
\hline Can f 4-PUR & 15 & 36 & 38 \\
\hline Can $\mathrm{f}$ 6-Purpurin & 14 & 33 & 51 \\
\hline Can f 6-PUR & 17 & 37 & 63 \\
\hline \multicolumn{4}{|c|}{ Perro-hámster } \\
\hline Can f 1-Phos 21 kDa & 17 & 31 & 60 \\
\hline Can f2-Phos $21 \mathrm{kDa}$ & 13 & 25 & 67 \\
\hline Can f 4-Pho s 21 kDa & 25 & 44 & 58 \\
\hline Can f 6-Phos 21 kDa & 21 & 41 & 64 \\
\hline
\end{tabular}

con el Ministerio de Salud de Colombia (www. minproteccionsocial.gov.co) y la Sociedad Protectora de Animales, alrededor de una de cada tres casas en el Valle de Aburrá tiene mascotas (perros $86 \%$ y gatos $17 \%$ ) y $23 \%$ tiene al menos dos mascotas de especies diferentes. Estos datos sugieren que hay una alta concentración de partículas derivadas de animales en el aire de Medellín. Sin embargo, no encontramos una relación directa entre la frecuencia de exposición a gatos o perros y sensibilización. Esto puede explicarse por la alta frecuencia de exposición indirecta en la escuela, el trabajo o el vecindario, que es difícil de cuantificar y, considerando los datos epidemiológicos comentados, ésta debe ser alta en esta región.
Cuadro 4. Identidad entre lipocalinas usando a gato como secuencia base

\begin{tabular}{lccc}
\hline Alineamiento & $\begin{array}{c}\text { Identidad } \\
\text { (\%) }\end{array}$ & $\begin{array}{c}\text { Núm. de } \\
\text { aminoácidos aminoácidos } \\
\text { idénticos }\end{array}$ & $\begin{array}{c}\text { Núm. de } \\
\text { similares }\end{array}$ \\
& \multicolumn{3}{c}{ Gato-bovino } \\
Fel d 4-Bos d 2 & 28 & 53 & 64 \\
Fel d 7-Bos d 2 & 19 & 38 & 54 \\
\multicolumn{5}{c}{ Gato-caballo } \\
Fel d 4-Equ c 1 & 68 & 130 & 30 \\
Fel d 7-Equ c 1 & 24 & 45 & 60 \\
& Gato-gallina & \\
Fel d 4-Purpurina & 16 & 35 & 57 \\
Fel d 4-PUR & 17 & 38 & 60 \\
Fel d 7-Purpurina & 17 & 36 & 50 \\
Fel d 7-PUR & 18 & 38 & 54 \\
& Gato-hámster & \\
Fel d 4-Pho s 21 kDa & 19 & 36 & 67 \\
Fel d 7-Pho s 21 kDa & 17 & 34 & 45
\end{tabular}

Otro factor importante que puede repercutir en la alta sensibilización a estos animales es la reactividad cruzada a la familia de proteínas lipocalinas. Entre las especies de perro, gato y caballo ${ }^{13}$ esta situación favorece la cosensibilización entre pacientes que tienen poca exposición a los animales. En nuestra población, esto podría explicar, al menos en parte, la alta cosensibilización a caballo, gato y perro. Con base en los análisis bioinformáticos, sugerimos que la cosensibilización podría ser por la alta identidad entre Can f 6, Fel d 4 y Equ c 1. De acuerdo con el patrón de sensibilización, todos los pacientes sensibilizados a caballo lo estuvieron también a perro, por lo que Equ c1 podría ser el principal alergeno de caballo. Por la reactividad cruzada Can $\mathrm{f} 6$ puede ser un importante alergeno para perro, pero no el único, porque la mayoría de los pacientes sensibilizados a perro no lo estuvieron a gato o caballo. Fel d 4 podría ser un alergeno importante para gato en nuestra población si se toma en cuenta la alta identidad que tiene con Can $f 6$ y la alta frecuencia de cosensibilización a perro entre los pacientes sensibilizados a gato; sin embargo, estas predicciones deben confirmarse 


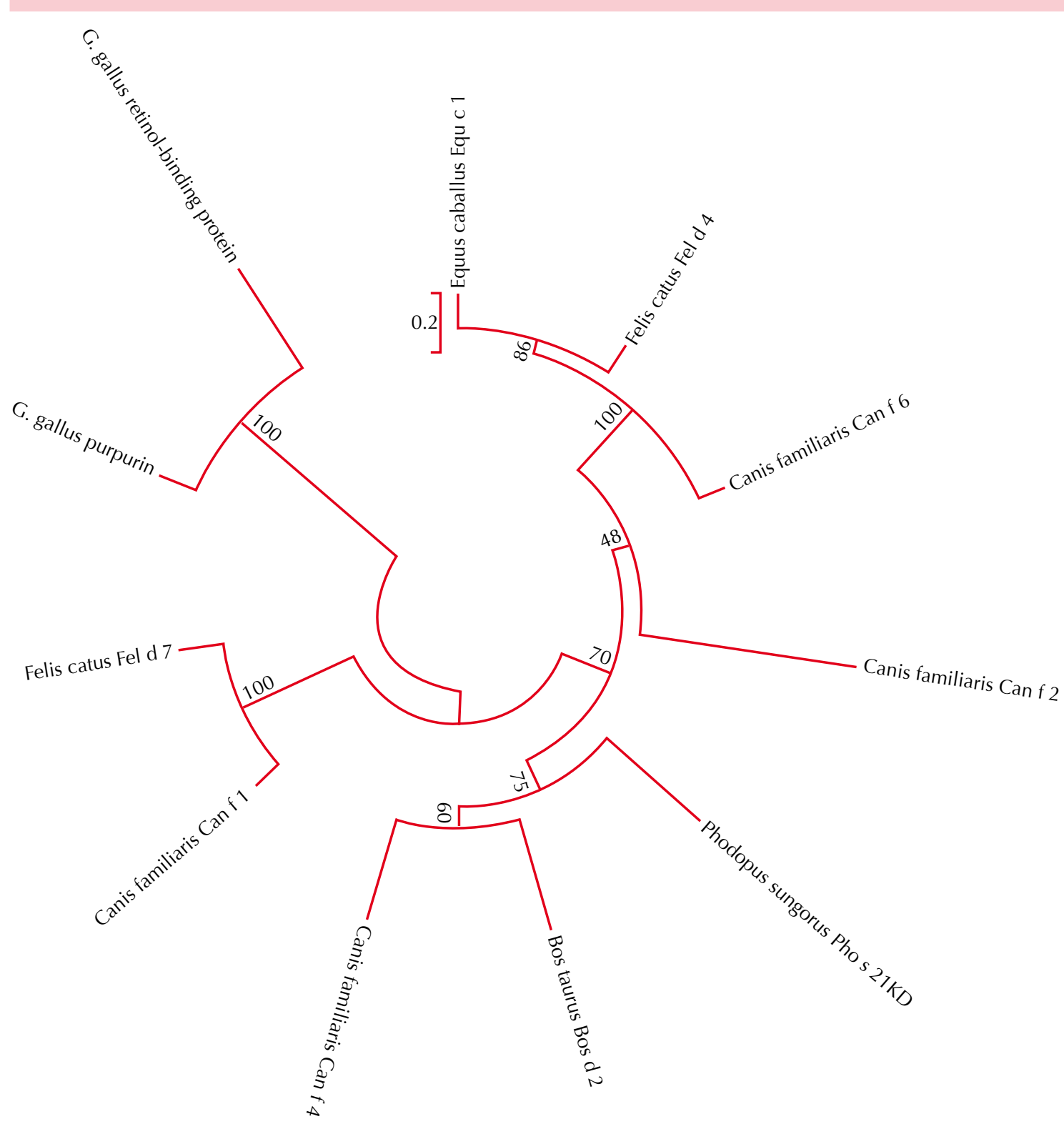

Figura 2. Árbol filogenético. El árbol óptimo con la suma de la longitud de la rama es igual a 5.00589491.

por métodos in vivo o in vitro. Nuestros resultados son similares a los encontrados por otros autores, lo que sugiere una alta probabilidad de reactividad cruzada, demostrada por análisis de inhibición y competición utilizando la técnica de ELISA entre Fel d 4, Can $\mathrm{f} 6$ y Equ c $1 . .^{14,15}$ De cualquier manera, la frecuencia de sensibilización a gato, perro y caballo es diferente entre las poblaciones, por lo que los factores del ambiente y las características genéticas de cada población influyen de manera importante en la prevalencia de la sensibilización a diferentes fuentes. 
Cuadro 5. Características generales de la población ( $\mathrm{n}=284)$

\begin{tabular}{lc}
\hline \multicolumn{1}{l}{ Datos generales } & Núm. (\%) \\
Sexo & \\
$\quad$ Mujer & $149(52)$ \\
$\quad$ Hombre & $135(48)$ \\
Fenotipos & \\
Asma & $159(56)$ \\
$\quad$ Rinitis & $237(83)$ \\
Conjuntivitis & $135(47)$ \\
Eccema & $71(25)$ \\
Edad (años) & Media 20 (límites: 1 a 71)
\end{tabular}

Cuadro 6. Características de la población por sensibilización $(\mathrm{n}=284)$

\begin{tabular}{lc}
\hline Datos generales & Núm. (\%) \\
Atopia & $227(80)$ \\
Perro & $136(48)$ \\
Gato & $27(9.5)$ \\
Pájaros* & \\
$\begin{array}{l}\text { Canarios, pericos, palomas y gallinas } \\
\text { Otros animales** }\end{array}$ & $33(14.5)$ \\
Caballo, conejo, hámster, cerdo & $7(7)$ \\
${ }^{*} \mathrm{n}=227 .{ }^{* *} \mathrm{n}=94$. &
\end{tabular}

Comparado con otras especies, se sabe poco acerca de la familia de lipocalinas y sus propiedades alergénicas en la gallina, vaca y hámster. ${ }^{16}$ Observamos una baja cosensibilización a hámster o vaca con gato o perro. Esta baja cosensibilización concuerda con la baja identidad entre las lipocalinas de estas especies y su mayor distancia filogenética. Existen pocos estudios acerca de la sensibilización a los pájaros..$^{5,17-21}$ Observamos que la frecuencia de sensibilización a pájaros como grupo fue mayor de $10 \%$, pero la sensibilización a gallina, paloma, periquito o canario separadamente fue menor de 5\%. ${ }^{5,22}$ Esta baja frecuencia de sensibilización, comparada con la de gato o perro, puede deberse a que pocas personas en nuestra población tienen pájaros como mascota, pero también puede deberse a la poca identidad con las lipocalinas de otras especies como gato o perro. La exposición directa parece tener mayor importancia en la sensibili- zación a pájaros que la reactividad cruzada a otras especies. Sin embargo, sólo tomamos las lipocalinas de gallina debido a que hasta nuestro conocimiento, son las únicas reportadas al momento, por lo que no podemos asegurar que la baja identidad observada con gato y perro se extienda a otras especies de pájaros.

Nuestro estudio tiene algunas fortalezas y limitaciones. Debido a que es un estudio in silico, las asociaciones encontradas entre los patrones de cosensibilización y la identidad de las lipocalinas nos permite explorar los alergenos que pueden tener mayor posibilidad de ser clínicamente relevantes en nuestra población, su efecto en la reactividad cruzada e identificar las posibles fuentes principales de sensibilización. Sin embargo, estos resultados, al ser predictivos, deben confirmarse con estudios in vitro para ratificar que los alergenos identificados son los causantes de la reactividad cruzada y las frecuencias de sensibilización encontradas; sin embargo, éste sería el primer paso para una búsqueda in vitro dirigida.

\section{CONCLUSIÓN}

De acuerdo con los análisis bioinformáticos y los patrones de sensibilización en una población del trópico, proponemos que algunas lipocalinas pueden tener un importante papel en la cosensibilización a gato, perro y caballo, pero no para hámster, vaca o pájaros. Fel d 4 y Equ c 1 podrían ser alergenos mayores para caballo y gato.

\section{Agradecimientos}

El Grupo de Alergología Clínica y Experimental (GACE) de la Universidad de Antioquia (MedeIlín, Colombia) financió este estudio.

\section{REFERENCIAS}

1. Dennis RJ, Caraballo L, García E, Rojas MX, et al. Prevalence of asthma and other allergic conditions in Colombia 20092010: a cross-sectional study. BMC Pulm Med 2012;12:17. 
2. Macías Weinmann A, Escamilla Weinmann C, Pazos Salazar NG, Valdés Burnes DA, González Díaz SN. [Sensitivity to animals' allergens in people working with animals]. Rev Alerg Mex 2010;57:185-189.

3. de Jong AB, Dikkeschei LD, Brand PL. Sensitization patterns to food and inhalant allergens in childhood: a comparison of non-sensitized, monosensitized, and polysensitized children. Pediatr Allergy Immunol 2011;22:166-171.

4. Burbach G, Heinzerling L, Edenharter G, Bachert C, et al. GA(2)LEN skin test study II: clinical relevance of inhalant allergen sensitizations in Europe. Allergy 2009;64:15071515.

5. Sánchez J, Diez S, Cardona R. Frecuencia de sensibilización a animales en un área tropical. Rev Alerg Mex 2014;61:81-89.

6. Brozek JL, Bousquet J, Baena-Cagnani CE, Bonini S, et al. Allergic Rhinitis and its Impact on Asthma (ARIA) guidelines: 2010 revision. J Allergy Clin Immunol 2010;126:466-476.

7. Santos MS, Alves MR, Freitas D, Sousa LB, et al. Ocular allergy Latin American consensus. Arq Bras Oftalmol 2011;74:452-456.

8. Hanifin JM. Diagnostic criteria for atopic dermatitis: consider the context. Arch Dermatol 1999;135:1551.

9. Heinzerling LM, Burbach GJ, Edenharter G, Bachert C, et al. GA(2)LEN skin test study I: GA(2)LEN harmonization of skin prick testing: novel sensitization patterns for inhalant allergens in Europe. Allergy 2009;64:1498-1506.

10. De Knop KJ, Verweij MM, Grimmelikhuijsen M, Philipse E, et al. Age-related sensitization profiles for hazelnut (Corylus avellana) in a birch-endemic region. Pediatr Allergy Immunol 2011;22(1 Pt 2):e139-49.

11. Matricardi PM, Bockelbrink A, Beyer K, Keil T, et al. Primary versus secondary immunoglobulin $E$ sensitization to soy and wheat in the Multi-Centre Allergy Study cohort. Clin Exp Allergy 2008;38:493-500.
12. Liccardi G, Salzillo A, Piccolo A, D'Amato G. Skin prick test to horse should be included in the standard panel for the diagnosis of respiratory allergy. J Investig Allergol Clin Immunol 2010;20:93-94.

13. Borres MP, Ebisawa M, Eigenmann PA. Use of allergen components begins a new era in pediatric allergology. Pediatr Allergy Immunol 2011;22:454-461.

14. Hilger C, Kuehn A, Hentges F. Animal lipocalin allergens. Curr Allergy Asthma Rep 2012;12:438-447.

15. Nilsson OB, Binnmyr J, Zoltowska A, Saarne T, et al. Characterization of the dog lipocalin allergen Can $f$ 6: the role in cross-reactivity with cat and horse. Allergy 2012;67:751-757.

16. Torres JA, de Las Heras M, Maroto AS, Vivanco F, et al. Molecular and immunological characterization of the first allergenic lipocalin in hamster: the major allergen from Siberian hamster (Phodopus sungorus). J Biol Chem 2014;289:23382-23388.

17. Chapman JA, Williams S. Aeroallergens of the southeast Missouri area: a report of skin test frequencies and air sampling data. Ann Allergy 1984;52:411-418.

18. Linna O, Niinimäki A, Mäkinen-Kiljunen S. Immunologic cross-reactivity between hen's feather and house-dustmite allergen extracts. Allergy 1994;49:795-796.

19. Kemp TJ, Siebers RW, Fishwick D, O'Grady GB, et al. House dust mite allergen in pillows. BMJ 1996;313:916.

20. Colloff MJ, Merrett TG, Merrett J, McSharry C, Boyd G. Feather mites are potentially an important source of allergens for pigeon and budgerigar keepers. Clin Exp Allergy 1997;27:60-67.

21. Kilpiö K, Mäkinen-Kiljunen S, Haahtela T, Hannuksela M. Allergy to feathers. Allergy 1998;53:159-164.

22. Sanchez J, Diez S, Cardona R. Sensibilización a aeroalergenos en pacientes alérgicos de Medellín, Colombia. Rev Alerg Méx 2012;59:139-147. 